Journal of mathematics and computer Science $\quad 8$ (2014) 28 - 32

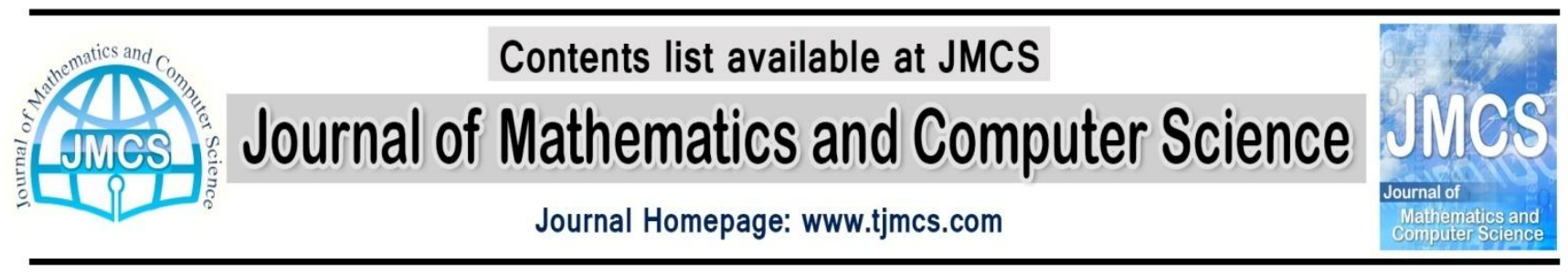

\title{
Simulation Method for Solving Stochastic Differential Equations with Constant Diffusion Coefficients
}

\author{
Behrouz Fathi Vajargah \\ Department of statistics, University Of Guilan, Rasht, Iran \\ fathi@guilan.ac.ir \\ Rahim Asghari \\ Departments of Applied Mathematics, University of Guilan, Rasht, Iran \\ meisam.mathhome@gmail.com
}

Article history:

Received February 2013

Accepted April 2013

Available online June 2013

\begin{abstract}
In the present paper a simulation method is presented for solving stochastic differential equations (SDEs). It was based on probability and statistics theory, so called random sampling or statistical test method. At the present paper, SDEs with initial conditions are considered. Some Numerical examples also are presented to confirm the efficiency.
\end{abstract}

Keywords: Stochastic differential equations, Simulation methods, Rejection method.

\section{Introduction}

Recently, because of wide range applications of stochastic differential equations (SDEs) in Finance, Physics, engineering, Biology, Aerospace and ..., attentions of researchers in many fields have been attracted. A (SDE) is a differential equation in which one or more of the terms are a stochastic process, resulting in a solution which is itself a stochastic process.

SDEs are used to model diverse phenomena such as fluctuating stock prices or physical system subject to thermal fluctuations. Typically, SDEs incorporate white noise which can be thought of as the derivative of Brownian motion (or the Wiener process).

A typical one-dimensional stochastic differential equation is of the form,

$d X_{t}=\sigma\left(X_{t}\right) d W_{t}+f\left(X_{t}\right) d t$

Where $d W_{t} \in R^{n}, n \in N$ is the differential of a $n$-dimensional Brownian motion, $R$ is space of Real numbers and $\mathrm{N}$ is the space of natural numbers. $\sigma(\bullet)$ an $\mathrm{dW}(\bullet)$ are functions on $\mathrm{R}$.

Numerical solution of stochastic differential equations is a young field relatively speaking. Almost all algorithms that are used for the solution of ordinary differential equations will work very poorly for 
SDEs, having very poor numerical convergence. One can find many different algorithms in Kloeden \& Platen (1995).

We categorized different numerical methods from the basic idea point of view into three main classes, Taylor series based methods in which one needs to evaluates derivatives and thus has limitations in application and difficulties in practical problems, Ronge Kotta based methods though are derivative free methods, they also have limitations in application because in these type of methods $f\left(X_{t}\right)$ should be smooth function satisfying lipschitz conditions in $X_{t}$ and finally Simulation based methods which are applicable to wide range of problems and do not have previous types Imitations. Simulation based methods mostly are based on Monte Carlo method, creatind sequence of random numbers and using them to test the stochastic system.

One of the most important simulation based methods is Rejection Sampling (RS) method which is recently used for solving ordinary differential equations. In the present paper RS based method is developed for solving SDE with constant diffusion coefficient

$d X_{t}=\sigma\left(X_{t}\right) d W_{t}+f\left(X_{t}\right) d t$

In which $\sigma($.$) is a constant number. We proposed a decision factor for these types of SDEs and present$ numerical examples in order to show the efficiency of the proposed method.

The present paper is organized as follows; we begin with stating a brief explaination on stochastic analysis and introducing rejection sampling technique in simulation that serves our purposes. At the next section the proposed method is introduced and finally numerical examples are presented in section 4.

\section{Statistical Analysis}

We describe a new algorithm for simulating a class of SDEs. It involves rejection sampling and when applicable returns exact draws from any finite-dimensional distribution of the solution of the SDE. Before describing the algorithm it was needed presenting some preliminaries and notations joint with the method.

Let $W=\left\{W_{t} ; 0 \leq t \leq T\right\}$ be a scalar Weiner process. Consider the general type of the onedimensional Ito diffusion:

$$
d X_{t}=f\left(t, X_{t}\right) d t+\sigma\left(t, X_{t}\right) d W_{t} \quad 0 \leq t \leq T, X_{0}=x \in R
$$

For drift coefficient $f:[0, T] \times R \rightarrow R$ and diffusion coefficient $\sigma:[0, T] \times R \rightarrow R$ under certain regularity conditions .It can be shown that (1) has a solution $\left\{X_{t} ; 0 \leq t \leq T\right\}$ weakly unique, that is, all the solutions have identical finite-dimensional distributions. Weak uniqueness relatively more general than path wise uniqueness is sufficient for simulation purposes.

\subsection{Rejection sampling method}

This method has been widely used in simulation techniques. It is frequently presented as follows. Assume that $F, G$ are probability densities with respect to (w.r.t) some measure on $R^{d}$ and that there exists $\varepsilon>0$ such that $\frac{F}{\varepsilon G} \leq 1$. Then the iterative algorithm is the following form:

\section{Rejection Sampling}

1. Generate $Y \sim G$.

2. Generate $U \sim \operatorname{Unif}(0,1)$. 
3. If $U<\frac{F}{\varepsilon G}(Y)$ return $Y$, else go to 1 .

Which returns an observation distributed according to $F$.

Let $(\mathrm{S}, \mathrm{S})$ be a sufficiently regular measurable space and $\nu, \mu$ probability measures on it such that $\mu$ is absolutely continuous with respect to (w.r.t.) $v$. Assume that there exists $\varepsilon>0$ such that

$\varphi:=\frac{d \mu}{\varepsilon d \gamma} \leq 1 \quad v$-a.s. and that it is easy to sample from $v$. The following proposition can be used to return draws form $\mu$.

Proposition1. (Rejection Sampling). Let $\left(Y_{n}, I_{n}\right) n \geq 1$, be a sequence of i.i.d. random elements taking values in $S \times\{0,1\}$ such that $Y_{1} \sim v$ and $P\left[I_{1}=1 \mid Y_{1}=y\right]=f(y)$ for all $y \in S$. Define $\tau=\min \{i \geq 1$ : $\left.I_{i}=1\right\}$. Then $P\left[Y_{\tau} \in d y\right]=\mu(d y)$.

This presentation of the Rejection Sampling scheme does not assume any order for the simulation of $Y$ and $I$ and, besides the certain conditional property given in the proposition, does not restrict in any other way the construction of the binary indicator $\mathrm{I}$.

Consider the stochastic process $X=\left\{X_{t} ; 0 \leq t \leq T\right\}$ determined as the unique solution of the SDE

$$
d X_{t}=f\left(t, X_{t}\right) d t+\sigma\left(t, X_{t}\right) d W_{t}, \quad 0 \leq t \leq T, X_{0}=x .(2)
$$

The drift function $f: R \times R \rightarrow R$ is presumed to satisfy the regularity properties that guarantees the existence of a global, weakly unique solution for (2). In particular, it suffices that $f$ is locally Lipchitz; that is, for each $M>0$ there exists $K_{M}>0$ such that

$$
|\alpha(y)-\alpha(x)| \leq K_{M}|y-x| \quad ; \quad|x| \leq M,|y| \leq M
$$

With a linear growth bound; there exists $K>0$ such that

$$
|\alpha(x)|^{2} \leq K^{2}\left(1+x^{2}\right), \quad x \in R .
$$

Since we are going to carry out rejection sampling it is convenient to think of the stochastic processes involved as measures induced on the space of continuous functions from $[0, T]$ to $R$. We denote by $\omega$ a typical element of this space. Consider the coordinate functions $W_{t}(\omega)=\omega(t), t \in[0, T]$, and the $\sigma$ field $C=\sigma\left(\left\{W_{t} ; 0 \leq t \leq T\right\}\right)$. To avoid confusion between the coordinate functions and the Brownian motion process, we use the generic notation $B M=\left\{B M_{t} ; 0 \leq t \leq T\right\}$ for a Brownian motion (BM) started at 0 . Let $w$ be the Wiener measure on $(C, C)$ so that $W=\left\{W_{t} ; 0 \leq t \leq T\right\}$ is a BM.

\subsection{Generating Random Numbers}

Generating random numbers is one of the most important parts of RS method. There are three main ways to generate random numbers, which were table of random numbers, physical method and pseudorandom numbers. Whereas, table of random numbers required a great deal of EMS memory, and the random numbers generated by physical method was unrepeatable. These two methods brought big trouble in practice applications. In random numbers generating, the most frequently used way was to use the pseudo-random numbers.

There are many ways to generate random numbers: additional congruential method, multiplicative congruential method, linear congruential generators (LCG) and mid-square method [6]. Here, we employed linear congenital generators as follows [7]:

$$
X_{i+1}=\left(a X_{i}+c\right) \operatorname{Mod} m, i=1,2, \ldots
$$




$$
R_{i}=\frac{X_{i}}{m}, i=1,2,3, \ldots
$$

Where $X_{i}$ is an arbitrary constant, $a, c$ and $m$ specified values, which is named as multiplier, increment and module respectively, $R_{i},(i=0,1, \ldots)$ are the random numbers we needed.

\section{Solution Approach}

In the present paper we are intended to solve SDE of the form (1) with constant diffusion coefficient; $\sigma\left(t, X_{t}\right)=1$. We introduce the decision factor as:

$$
F \leq \varepsilon G
$$

In which $G, F$ will considered as $G=\frac{f(t, x) d t+d w}{x}$, and $F=\frac{d x}{x}$.

Remark 1: As $x$ falls in the neighborhood of zero numerical error increases, especially when the answer is near to zero or with initial condition zero, this will create a large error. In order to avoid this error one simple suggestion is to transform the state far from zero and then after solution transform it back. This transformation should be such that the minimum numerical error to be occurs.

\section{Numerical examples}

In order to show the efficiency, we present some numerical examples which was solved using proposed method and Euler's method

Example 1. Consider very simple form of the (1) with $\sigma\left(t, X_{t}\right)=1, f(t, x)=0$.

In figure1, numerical simulation of example1 (blue stars) compared with Euler method (red stars).

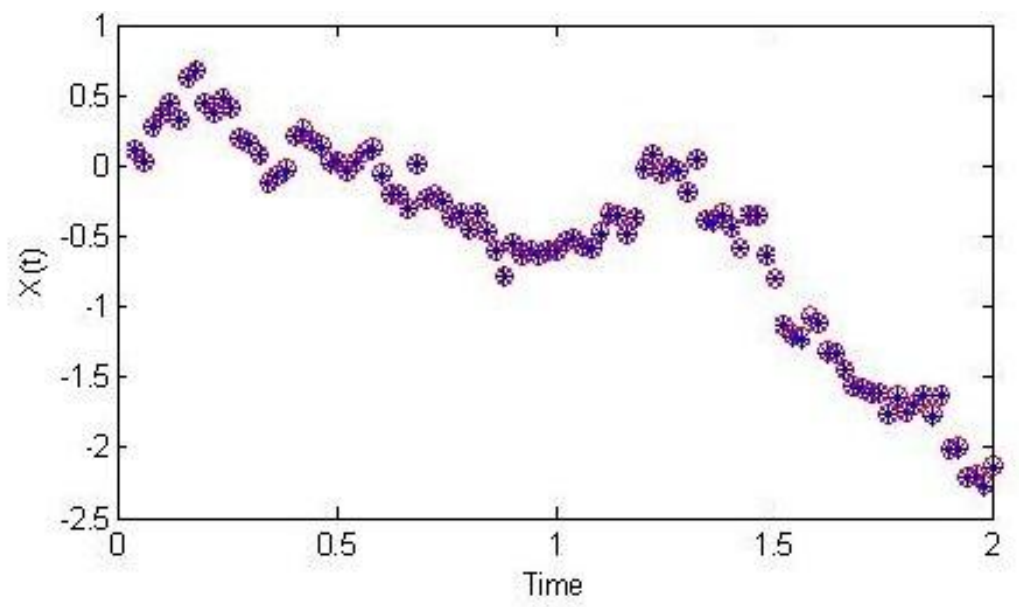

Figure1

Example 2. Consider stochastic differential equation

$$
d y=(-y+x+1) d x+d w
$$

with initial condition $y(0)=0$.

This problem is solved via the presented method, and compared with Euler method. 


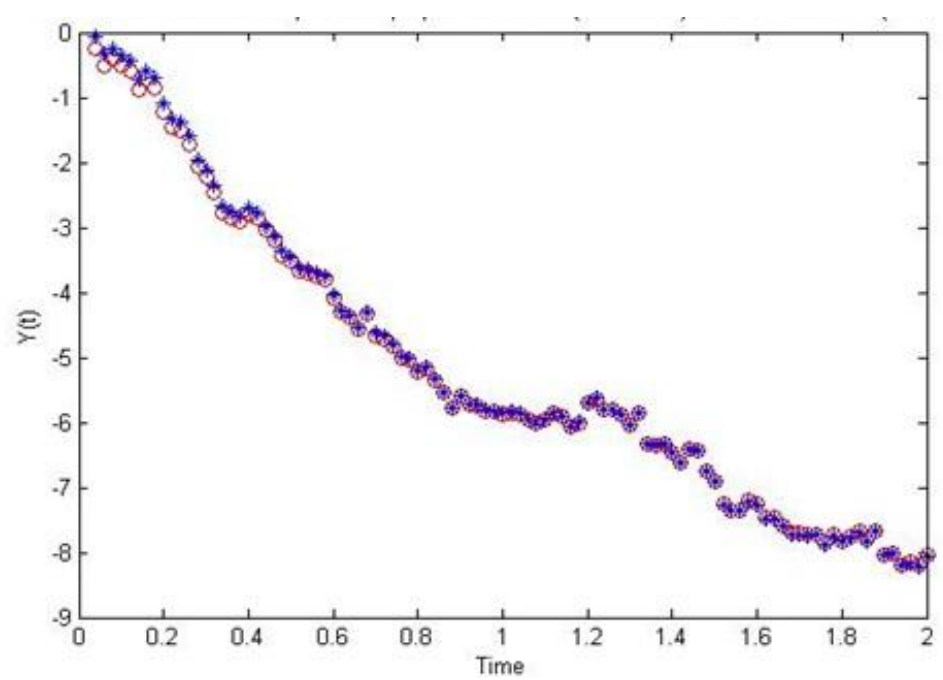

Figure2

\section{Conclusion}

In the present paper RS simulation technique is implemented for solving special form SDEs. As mentioned proposed method is solved this for of SDE with high accuracy. The proposed method is the extension of the [4] method for SDEs. The way of generation random numbers in addition to the ways presented in [4] has effects on accuracy.

\section{References}

[1] P.E. Kloeden and E. Platen, Numerical Solution of Stochastic Differential Equations, Springer (1995). [2] K. Burrage, P. M. Burrage and T. Tian, Numerical methods for strong solutions of stochastic differential equations: an overview, Proc. R. Soc. Lond (1995).

[3] Chien-Cheng Chang, Numerical Solution of Stochastic Differential Equations with constant diffusion coeffcient, mathematics of computation, vol 49, p 523-542, (1987).

[4] W.Zhong, Z. Tian, Solving Initial Value Problem of Ordinary Differential Equations by Monte Carlo Method, Northwest Inst. of Nucl. Technol, (2577 - 2579), 26-28 July (2011)

[5] A. BESKOS AND G. O. ROBERTS, EXACT SIMULATION OF DIFFUSIONS, The Annals of Applied Probability, Vol. 15, No. 4, 2422-2444, (2005).

[6] Oksendal, B. K. Stochastic Differential Equations: An I ntroducti on with Applications. Springer, Berlin (1998).

[7] S.Y. Xu. The Application of Monte Carlo Method in Nuclear Physics Experiment (Revised Edition) (in Chinese). Beijng: Atomic Energy Press. (2006). 